\title{
Germanica
}

\section{Versuche zur Schaffung einer Gegenöffentlichkeit in den westdeutscheb Hörspielen der fünfziger Jahre}

Les pièces radiophoniques ouest-allemandes dans les années cinquante

Jonas Tophoven

\section{OpenEdition}

Journals

Édition électronique

URL : http://journals.openedition.org/germanica/2215

DOI : $10.4000 /$ germanica.2215

ISSN : 2107-0784

\section{Éditeur}

Université de Lille

\section{Édition imprimée}

Date de publication : 1 décembre 1994

Pagination : 91-106

ISSN : 0984-2632

Référence électronique

Jonas Tophoven, «Versuche zur Schaffung einer Gegenöffentlichkeit in den westdeutscheb Hörspielen der fünfziger Jahre », Germanica [Online], 14 | 1994, Online erschienen am: 30 September 2013, abgerufen am 06 Oktober 2020. URL : http://journals.openedition.org/germanica/2215 ; DOI https://doi.org/10.4000/germanica.2215

Ce document a été généré automatiquement le 6 octobre 2020.

(c) Tous droits réservés 


\title{
Versuche zur Schaffung einer Gegenöffentlichkeit in den westdeutscheb Hörspielen der fünfziger Jahre
}

\author{
Les pièces radiophoniques ouest-allemandes dans les années cinquante
}

\author{
Jonas Tophoven
}

1 Der Themenkomplex Literatur und Zeitgeschehen verdient es, auf die Problematik des Hörspiels übertragen zu werden, weil Zeitgeschehen als Aktualität ein Produkt der Medien, insbesondere des Rundfunks ist, und die Literatur in Form von Hörspielen nicht der Aktualität gegenübersteht, sondern sich desselben Mediums für eigene Zwecke zu bedienen sucht. Man kann sich dabei die Frage stellen, ob eine solche Standortverschiebung der Literatur einen Einfluß auf die Art und Weise hat, wie Zeitgeschehen reflektiert wird.

2 Brechts Lindberghflug ${ }^{1}$ aus dem Jahre 1929 ist nur eines der zahlreichen Beispiele für die Gestaltung unmittelbarer Zeitgeschichte durch die Hörspielautoren der Weimarer Republik. In seiner Untersuchung des Hörspiels im Dritten Reich ${ }^{2}$ erwähnt Wolfram Wessels den Protestbrief eines Hörers gegen die Manie, Anfang der dreißiger Jahre Nachrichten im kürzestmöglichen Abstand hörspieldramatisch zu verwerten. Die Tendenz wird $1940 \mathrm{im}$ Rahmen des Propagandarundfunks auf die Spitze getrieben. Gerd Eckert, Pionier unter den akademischen Experten des Hörspiels und eifriger Verfechter des nazistischen Propagandahörspiels, beschreibt die unmittelbare Umgestaltung des Zeitgeschehens folgendermaßen.

Am Abend des 7. Februar (1940) konnte man in den deutschen Zeitungen davon lesen, daß die Iren Peter Barnes und James Richards in Birmingham hingerichtet worden waren für ein Verbrechen, das man ihnen nicht bewiesen hatte und das sie bis zum letzten Atemzug abstritten. 24 Stunden später brachte bereits der deutsche Rundfunk ein Hörspiel unter dem Titel «Das Todesurteil von Birmingham», das in dramatisch wirksamem Aufbau jeden Hörer das Schicksal der beiden Iren miterleben ließ. Wie war dieses Hörspiel entstanden? Kaum war die Nachricht von 
der vollzogenen Hinrichtung bekanntgeworden, erhielt ein Schriftsteller den Auftrag, mit Hilfe ausführlicher Unterlagen, die ihm zur Verfügung gestellt wurden, unter dem Eindruck dieses jüngsten Geschehnisses in wenigen Stunden ein Manuskript zu schreiben. Dieses kam dann ins Rundfunkhaus, und hier begann eine Gemeinschaftsarbeit, die allein die Aufführung dieses Hörspiels möglich machte ${ }^{3}$.

3 Der sogenannte «totale Zusammenbruch» brachte dem Rundfunk eine größere politische Unabhängigkeit und eine nun systematisch angewendete Aufzeichnungsmöglichkeit: das Tonband. Damit waren die Voraussetzungen geschaffen für Hörspiele literarischer Qualität. Das Tonband hat ein wesentliches Gestaltungselement der Hörspieldramaturgie, die Blende, zwar nicht hervorgebracht, aber dessen Anwendung erleichtert. Außerdem wurde der Schnitt als Kontrast zur Blende möglich. Schnitt und Blende stehen im Gegensatz zur Kontinuität des Zeitgeschehens. Man könnte also meinen, daß das Hörspiel dazu einlädt, Zeitgeschehen besonders von seiner linearen Form her umzuwandeln. Doch das gilt eher für neuere Hörspiele.

4 Ich möchte mich hier dagegen auf einige Hörspiele der fünfziger Jahre beschränken. Diese Zeit gilt gemeinhin als die Blütezeit des literarischen Hörspiels, des Hörspiels als Wortkunstwerk.

5 Die Situation ist damals besonders günstig:

- Die von den Allierten erzwungene Entwicklung des UKW-Netzes ${ }^{4}$ führt letztendlich zu einer wesentlichen Verbesserung der Empfangsqualität.

- 1952 gibt es in der BRD 10 Millionen Rundfunkteilnehmer, die noch wenig andere Unterhaltungsmöglichkeiten besitzen.

- Im öffentlich-rechtlichen Monopol verfügen die regionalen Sender über geregelte Einkünfte, zahlen gute Honorare und sind bemüht, mittels des Hörspiels eine neue literarische Form zu schaffen, die den Rundfunk kulturell legitimiert.

Der Rundfunk kann in dieser Zeit auf einige routinierte Hörspielautoren zurückgreifen, die ihr Handwerk in der Weimarer Zeit oder gar während der Nazizeit erlernten ${ }^{5}$. Pro Jahr kommen damals etwa 120 literarische Hörspiele zur Ursendung, und tatsächlich haben damals fast alle namhaften Autoren Hörspiele geschrieben. Und viele haben, zumindest zeitweilig, diese Form ernstgenommen. So wird berichtet ${ }^{6}$, daß die Gruppe 47 bei ihrem Herbsttreffen vom Jahre 1953 fast ausschließlich Hörspielmanuskripte vorgelesen hat. Verlage druckten häufig Hörspiele, die auch in Buchform Absatz fanden. Die Brücke vom Hörspiel als einer eigenständigen radiophonen Gattung zur Verlagsliteratur war damals leicht $\mathrm{zu}$ schlagen. Allerdings wurde die künstlerische Relevanz dieser zumindest in formaler Hinsicht literarischen Hörspiele in den sechziger und siebziger Jahren angezweifelt. Schon in den fünfziger Jahren warf man ihnen ausgerechnet ihren mangelnden Zeitbezug vor. Heinz Schwitzke, der langjährige Chefdramaturg des NWDR ${ }^{7}$, schreibt dagegen in seiner 1963 erschienenen Dramaturgie und Geschichte des Hörspiels ${ }^{8}$ :

Eher ist verwunderlich, daß - obwohl seit 1951 immer von den Träumereien im Hörspiel geredet wird, die die Gegner «Gespensterspuk» schimpfen - dennoch soviel Zeitgeschichte und Zeitwirklichkeit in die Hörspiele eingeflossen ist. Man kann von kaum einer anderen Kunstform sagen, daß etwa jedes zweite Werk Aktualität repräsentiert ${ }^{9}$.

7 Dieser besondere Zeitbezug wurde aber in den neueren hörspielgeschichtlichen Studien wie die von Reinhard Döhl ${ }^{10}$ und Margaret Bloom ${ }^{11}$ nicht besonders hervorgehoben. Wenn man in der damaligen Sekundärliteratur blättert, stößt man 
allenfalls auf einen Aufsatz von Oskar Wessel über Aktualität im, der 1954 in der Fachzeitschrift Rundfunk und Fernsehen erschien. Wessel wurde 1947 Preisträger des ersten. Hörspielpreisausschreibens des NWDR, und zwar mit dem Hörspiel Hiroshima: es beschrieb das Leben der Stadt kurz vor dem Abwerfen der Bombe. 1948, also knapp drei Jahre nach dem Ereignis. Das Thema Hiroshima hat viele Autoren der Nachkriegszeit beschäftigt ${ }^{12}$. Kann man aber später noch im engen sinne von Reflexion des Zeitgeschehens reden, oder handelt es sich dann eher um die Behandlung eines schon historisch gewordenen Stoffes? Für die zahlreichen Hörspiele der sogenannten «Vergangenheitsbewältigung» kann man sich die gleiche Frage stellen. Manfred Haeberlens Bearbeitung von Plieviers Roman Stalingrad, gesendet im Jahre 1948 in Baden-Baden, zeichnet sich wie Borcherts Draußen vor der Tür aus dem Jahre 1947 durch scharfe Kritik an den Wehrmachtsoffizieren aus. In der Neuinszenierung von Borcherts Stück, Ende der fünfziger Jahre, wurden einige Passagen gestrichen, weil die Offiziere nicht zu schlecht wegkommen sollten. Auf diese Weise verwandelt sich die nötige Verarbeitung der Vergangenheit in die von den Kritikern angeprangerte Ablenkung von der Gegenwart.

Die «Vergangenheitsbewältigung» erfolgte auch nicht unbedingt über die historische Thematisierung des Zeitgeschehens. So soll 1950 Otto Heinrich Kühners Stück Die Übungspatrone $e^{13}$ stark auf die Zuhörer gewirkt haben. Ob die Geschichte im Zweiten Weltkrieg tatsächlich stattgefunden hat, ist nebensächlich: Soldaten eines Erschießungskommandos trösten sich beim Gedanken, daß einer von ihnen vielleicht die Übungspatrone abgefeuert hat. Es stellt sich aber heraus, daß einer der Soldaten nicht geschossen hat, und die Übungspatrone sich gerade in seinem Gewehr befand. Ein solches Sinnbild vermag es, das Gefühl von Reue und Verantwortung zu erwecken, ohne daß der Hörer der Nostalgie geschichtlicher Erlebnisse zu verfallen droht. Als Erich Kuby 1951 dagegen gezielt das nazistische Kriegstreiben in Brest anprangerte ${ }^{14}$, trug es ihm eine Verleumdungsklage ein.

Die Ausgrenzung des tatsächlichen Zeitgeschehens, die man in den Hörspielen der fünfziger Jahre feststellen kann, ist auch formal bedingt. Der Rundfunk grenzt die Fiktion von den Nachrichten ab, um nicht den kommunikativen Konsens zu gefährden. Diese Abgrenzung wird selbst im besonderen Stil der Inszenierungen deutlich: Heinz Schwitzke schreibt über den «tonangebenden» Regisseur der Zeit:

Schröder-Jahn war in der Tat der Erfinder jener Methode, bei der plötzlich alles, was bis dahin galt, ins Gegenteil verkehrt wurde: Stille, das war nicht mehr die Lücke zwischen Worten und Tönen, sondern Worte und Töne schwammen vereinzelt und vorsichtig in dem Meer der Stille, das sie trug ${ }^{15}$.

10 Hinzu kommt der Legitimitätsdruck, dem das Hörspiel als neue literarische Gattung ausgesetzt ist. Und nicht zuletzt die Tatsache, daß der intellektuelle Zeitgeist damals stark von Sartre und Adorno geprägt war. Auf der einen Seite die existentialistische Herausforderung, der Autoren gerade als Autoren gerecht zu werden glaubten; auf der anderen die Vorstellung autonomer Kunst, die alleine in der Lage wäre, die spätbürgerliche Kultur zu überdauern.

11 Allerdings nimmt der in literarischen Kreisen gepflegte Nonkonformismus zumindest bei einigen Mitgliedern der Gruppe 47 bisweilen einen politischen Charakter an. Die Mitglieder haben anfangs Bedenken gegen eine Mitwirkung beim Rudfunk. Ihre politische Opposition wird sich im Laufe der fünfziger Jahre an der Frage der Neuschaffung eines deutschen Heeres (und ihrer atomaren Bewaffnung) entzünden. 
Diese politische Grundhaltung bedingt die Art, wie die Autoren das Zeitgeschehen behandeln. Gegen das Vergessen, gegen die Verharmlosung, so kann man zumindest die Hypothese aufstellen, verfolgen sie die Absicht, im CDU-Staat eine Art Gegenöffentlichkeit zu bilden.

Beim Versuch, das Verhältnis von Zeitbezug und Gegenöffentlichkeit anhand von drei Hörspielen zu durchleuchten, werde ich mich noch eines dritten Begriffes bedienen : die Thematisierung der medialen Kommunikation.

Die Hypothese lautet: Wenn der literarische Grad eines Werks mit dem Grad seiner Gestaltung $\mathrm{zu}$ tun hat, dann bedingt die Thematisierung des Zeitgeschehens die Thematisierung der medialen Kommunikation. Mit anderen Worten: Der literarische Charakter eines Hörspiels wird nicht durch seine Autonomie gegenüber dem Zeitgeschehen gewährleistet, sondern dadurch, da das Zeitgeschehen zum Zwecke einer gegenöffentlichen Wirkung mittels einer Thematisierung der medialen Kommunikation hervortritt.

Günter Eichs Träume aus dem Jahre $1950^{16}$ sind in dieser Hinsicht geradezu paradigmatisch. Vielerorts als Geburtsstunde des deutschen Hörspiels gefeiert, wird an ihnen der gegenöffentliche Impuls wie kaum woanders deutlich. Das Abschlußgedicht mit den Sätzen «Seid nicht öl, seid Sand im Getriebe der Welt» ist allgemein bekannt, und so schnell sich in den fünfziger Jahren das sogenannte Eich-Maß herausbildete, so sehr war es mit diesem gegenöffentlichen Impuls verbunden.

Reinhard Döhl weist in einem Aufsatz über die Hörspielphilologie ${ }^{17}$ darauf hin, daß dieser Satz nicht im ursprünglichen Sendemanuskript steht. Er soll also von Eich für den ersten Sammelband seiner Hörspiele ${ }^{18}$, der bezeichnenderweise den Titel «Träume» trägt, hinzugefügt worden sein. Als gründlicher Hörspielphilologe überprüft Döhl die Tonbandaufzeichnung und stellt fest, daß diese sehr wohl den berühmten Schlußsatz enthält. Seine Erklärung lautet, daß dieser Zusatz später aufgenommen, und der Originalaufnahme hinzugefügt worden ist. Die ungewöhnliche Entrüstung, die dieses Hörspiel bei den Hörern hervorrief, wäre demnach nicht auf diese Aufforderung zum Widerstand zurückzuführen.

Mag sein, daß die fünf Szenen eher wegen ihrer aussichtslosen Grausamkeit schockierten. Hermann Naber $^{19}$ hat hervorgehoben, wie eng Eichs Träume mit der Realität verbunden sind. Der erste Traum ist die grausam-absurde Zuspitzung der Situation, die dreißig Millionen Europäer als «displaced persons» oder Flüchtlinge im Laufe der vierziger Jahre kennengelernt haben. Der zweite Traum nimmt Bezug auf die drohende Bevölkerungsexplosion und stellt einen Zustand dar, wo Kinder anderen Menschen zum Fraß verkauft werden. Der Traum des australischen Automechanikers Lewis Stone verweist auf die Bedrohung, der dieses Land durch Japan im Laufe des Zweiten Weltkriegs ausgesetzt war. Der Feind drohte jederzeit, heranzurücken und die Einwohner in unwillkommene Flüchtlinge zu verwandeln. Lediglich bei den letzten beiden Träumen fällt der Verweis auf historische Zusammenhänge etwas schwerer.

Im ersten Sendemanuskript soll der Autor durch ein Rahmengedicht den Zeitbezug des Hörspiels hervorgehoben haben. Laut Döhl beginnt es mit «vier prosaischen Sprecherhinweisen auf den Atomversuch von Bikini 1946, auf die Zunahme der Mißgeburten seit der Bombardierung von Hiroshima und Nagasaki, auf Wasserstoffbombe und bakterielle Kriegsführung» ${ }^{20}$. 

Thematisierung der medialen Kommunikation. So unterstreichen die kurzen Einleitungen zu den Träumen deren Gegensatz zu herkömmlichen Nachrichten: In der Nacht vom 1. zum 2. August 1948 hatte der Schlossermeister Wilhelm Schulz aus Rügenwalde in Hinterpommern, jetzt Gütersloh in Westfalen, einen nicht sonderlich angenehmen Traum, den man insofern nicht ernstnehmen muß, als der inzwischen verstorbene Schulz nachweislich magenleidend war. Schlechte Träume kommen aus dem Magen, der entweder zu voll oder zu leer ist ${ }^{21}$.

Abgesehen von den Zeitbezügen, die durch die ironische Formulierung hindurchscheinen, haben wir es hier mit einer regelrechten Antinachricht zu tun.

Das Rahmengedicht fungiert nicht nur als Bindeglied zwischen den einzelnen Träumen, sondern als Vermittler zwischen ihrer Vorstellung und deren Inhalt. Das Gewicht wird dabei deutlich zuungunsten einer Verharmlosung verschoben: Eich schreibt zum Beispiel: «Denke daran, da der Mensch des Menschen Feind ist und da er sinnt auf Vernichtung»... «Denke daran, wenn eine Hand dich zärtlich berührt, denke daran in der Umarmung deiner Frau...» Wie die wütenden Proteste der Hörer nach der ersten Sendung, so ist die pathetische Verschärfung des Tons in der Druckfassung nicht unbedingt ein Beweis für literarische Relevanz. Marshall Mac Luhan hat die Verbindung aufgezeigt, die zwischen der Erfindung des Telegrafen und der Entwicklung des modernen britischen Krankenhauswesens bestand ${ }^{22}$. Eich hebt hier einen Zustand der universalen Verantwortung hervor, der latent vorhanden ist. Aber gleichzeitig verharmlost er am Ende des Textes den Empfang von Nachrichten durch die deutschen Zuhörer. Die Thematisierung der medialen Kommunikation ist also widersprüchlich und entspricht dem Schwanken des Werkes zwischen der Hörspielund der Buchform.

In Biedermann und die Brandstifter reflektiert Max Frisch die mediale communikation auf konsequentere Weise. Das Hörspiel ist eine Auftragsarbeit aus dem Jahre 1952 und kommt 1953 in München zur Ursendung. Die Fabel ist schon im Tagebuch 1946-49 enthalten. Ab 1958 wird der Stoff als Bühnenstück zum Welterfolg. Frisch verzichtet noch nicht auf jegliche Rahmenhandlung, aber der Sprecher als Erzähler stellt sich der Person Biedermann als Verfasser vor. Dieser Verfasser gibt sich wie die üblichen Erzähler als ein Erklärer und Wegweiser, der in die Handlung einführt und Szenen miteinander verknüpft. In Wirklichkeit fungiert die Präsenz des Verfassers aber als intendierter Stör faktor. Wie kaum in einem anderen Stück dieser Zeit werden die künstlerischen Konventionen aufgebrochen. Stimmen verweisen mal auf Sprecher, mal auf Figuren. Scheinrealitäten werden auf- und abgebaut. Frisch bezieht sogar den üblichen Schlußkommentar in die Hör Spielgestaltung hinein.

Biedermann und die Brandstifter ist ein Versuch, episches Theater auf die innere Bühne des Hörspiels zu verlegen. Ein Versuch, der zuweilen seine theatralische Herkunft verrät.

Viel deutlicher als in der späteren Bühnenfassung geht Frisch hier direkt mit den deutschen Hörern ins Gericht. 1949, im Aufsatz Kultur als Alibi ${ }^{23}$, hatte Frisch eine schon im Tagebuch formulierte Kritik an den Deutschen wieder aufgegriffen. Einer abgehobenen ästhetischen Kultur, die Virtuosen wie Heydrich hervorbringe, sei die schweizerische Auffassung der Kultur als bezogen auf staatsbürgerliche Leistungen entgegenzustellen. 


\section{Wirklich}

Im Hörspiel setzt der Verfasser sich in Szene, aber er läßt auch Biedermann als deutschen Widerpart zu Wort kommen. Etwa in der Mitte des Stücks wirft dieser Biedermann seinem Verfasser vor, es sich zu leicht gemacht zu haben: hinterher wisse man doch alles besser. In der Tat spielt Frisch mit seiner eigenen ambivalenten Haltung gegenüber den Deutschen. Am Anfang benutzt der Verfasser noch das «wir»: der Verfasser zu Biedermann:

Wir sind bereit, nicht bloß den Urhebern unserer Katastrophe eine volle Amnestie zu gewähren, sondern sogar uns selbst.... ${ }^{24}$

Und etwas weiter:

Wie sollen wir ein neues Seldwyla erbauen ohne Sie?25 als Zwischenform der atomaren und der totalitären Bedrohung zu wählen. Im Spiel als solchen werden Zeitbezüge tunlichst vermieden, aber der Hörer wird indirekt dazu veranlaßt, die Bezüge selber herzustellen. Und zwar ist die Eingangsrede des Verfassers an «die lieben Hörerinnen und Hörer» so formuliert, daß diese sich mit Biedermann als dem Überlebenden identifizieren müssen. Der Verfasser betont, daß er eine erfundene Katastrophe gewählt habe, um Emotionen zu vermeiden. Dadurch steht außer Zweifel, daß der Brand von Seldwyla auf den Komplex Nazizeit, Krieg und Zerstörung verweist. Dieser Eindruck wird durch die schon erwähnten Äußerungen des Verfassers über die volle Amnestie und den Bedarf an allen Kräften, um ein neues Seldwyla aufzubauen, bekräftigt.

Trotzdem fällt es schwer, die fiktive Handlung mit den Ereignissen der jüngsten Vergangenheit in Beziehung zu setzen. Die Flucht in die Abstraktion, die man auch in vielen anderen Hörspielen der fünfziger Jahre antrifft, wird in der späteren Bühnenfassung noch verschärft. Das Lehrstück verliert dann bekanntlich seine epischen Brechungen zugunsten von Choreinlagen, die Frischs Dramaturgie in den Bannkreis von Dürrenmatt rücken.

29 Aber kommen wir noch einmal kurz auf die vergessene Hörspielfassung zurück: Nehmen wir an, Frischs Projekt hätte tatsächlich darin bestanden, dem deutschen Hörer zu zeigen, daß er die Katastrophe ermöglicht hat. Liegt die Verfasserfigur falsch, wenn sie behauptet, daß eine Darstellung der Zeitgeschichte diesen Zweck nicht erreichen könnte?

Schauen wir uns das Alternativprogramm des Verfassers näher an. Auf der einen Seite optiert er für eine erfundene Geschichte. Schon der Name Seldwyla steht für Fiktion. Die Geschichte ist nicht nur erfunden, sondern glatt unwahrscheinlich - die Verfasserfigur trägt Biedermann aber auf, besonders glaubwürdig zu spielen. Die letzten Sätze, die die Verfasserfigur ausspricht, bevor die eigentliche Handlung beginnt, sind allerdings etwas verwirrend:

...unser Hörer soll das Gefühl haben: Ein guter und anständiger Mensch, dieser Biedermann, eine Seele von Mensch. Also das Gefühl: Hand aufs Herz, so hätte ich auch gehandelt! Nur dann werden wir finden, Herr Biedermann ist unschuldig; er tut ja nur, was wir alle tun. Und nur dann, wenn von Verantwortung nicht die Rede sein kann, sind wir bereit, zu vergessen, wie es zu dieser Katastrophe (in Seldwyla) gekommen ist - und bereit für die nächste ${ }^{26}$. 

einen mimetischen Vorgriff auf die Gestaltungsperspektive, um eine « mise en abyme ». Man kann die letzten Sätze aber auch in einem programmatischen Sinne lesen: die Vorgabe an den Sprecher, Biedermann besonders wahrscheinlich und als Identifikationsfigur darzustellen, ist durch die Vorgabe der erfundenen Geschichte nicht realisierbar. Das illusionistische Hörspiel der fünfziger Jahre läuft hier gegen eine Wand. Frischs Trick ist dabei die Mischung unvereinbarer Spiel- und Illusionsebenen, welche gerade dadurch auf die Realität zurückverweisen.

weit zur «Vergangenheitsbewältigung». Wie wird aber das unmittelbare Zeitgeschehen sonst reflektiert? Späte Freilassungen aus russischer Gefangenschaft, der 17. Juni 1953, der innerdeutsche Ost-West Konflikt, die Wiederbewaffnung, das Berlinultimatum... all diese Ereignisse werden kaum in namhaften Werken aufgegriffen.

on Schwitzke hervorgehoben wurde das Hörspiel Früher Schnee am Flu $\beta$ von Heinz Huber $^{27}$ : ein Journalist berichtet der indifferenten Öffentlichkeit von den Umständen der Hinrichtung eines koreanischen Freudenmädchens, das verdächtigt wird, eine Partisanin zu sein. $\mathrm{Zu}$ nennen wäre natürlich auch Wolfgang Weyrauchs Hörspiel Die japanischen Fischer ${ }^{28}$. In naiv verfremdeter Sprache ist dort von der Verseuchung japanischer Fischer durch die Auswirkungen eines überirdischen Atombombentests die Rede. doch in großer geographischer Ferne.

Die fünf Sekunden des Mahatma Gandhi (Walter Erich Schäfer, 1949), Die Minute des Negers (Wolfgang Weyrauch, 1954), und 1960 Die Nacht der Giraffe... Der Titel von Anderschs 1960 urgesendeten Hörspiels klingt wie eine Tarnung des brisanten Stoffes. Nach eigener Aussage beginnt der Autor unmittelbar nach de Gaulles «Machtergreifung» vom Mai 1958 mit der Arbeit an einem Hörspiel, das vermuten läßt, de Gaulle habe den Algier-Putsch durch Mittelsmänner selber angezettelt. Andersch verbindet wahre und erfundene Elemente in einer Momentaufnahme vom Abend des 29. Mai 1958. Die historischen und politischen Ereignisse um de Gaulle werden dem Treiben des Quartier Latins gegenübergestellt. Der Autor baut Zitate des Publizisten Jean-Jacques ServanSchreiber ein, erwähnt den Dramatiker Artur Adamov, den Pariser Buchhändler Flinker. Hinter Fayard verbirgt sich wahrscheinlich Hubert Beuve-Méry, von der kleinen Buchhandlung des Philosophen Mondello in der rue du Dragon blickt man auf die Editions de Minuit ${ }^{29}$.

Zeitgeschehen nimmt aber sogleich den Charakter von Geschichte an, ja die Mischung von verschiedenen Gestaltungsebenen verleiht dem Geschehen einen fast mythischen Charakter: nebenan, in Frankreich, ist was los! Da ist Krieg, da lauert Gefahr, da gibt es historische Persönlichkeiten. Widerholt vergleicht Andersch die französische Gegenwart mit den Religionskriegen des 16. Jahrhunderts. Der Reporter, als Sprachrohr für den inneren Monolog des Generals, sagt:

Ich werde mit den Fraktionen, mit der Liga und den Hugenotten spielen wie mit Bällen ${ }^{30}$.

Andersch scheint fiktive und lyrische Elemente einfließen zu lassen, um die Aussage des Hörspiels zu verharmlosen, um es sendbar zu machen. Bezug auf die Begebenheiten der medialen Kommunikation nimmt der Autor auch in der Dreiteilung des 
Kommentars. Zwischen den Szenen kommen in gleicher Reihenfolge ein Erzähler, ein Reporter und ein Dichter immer hintereinander zu Wort. Mag sein, daß der FeatureAutor sich dabei auf sein Handwerk besinnt. Aber der Reporter berichtet vom inneren Monolog de Gaulles, der Erzähler beschreibt die Demonstration im Quartier Latin wie ein Reporter. Und der Dichter scheut sich nicht vor zeitgeschichtlichen Bezügen, ganz abgesehen davon, daß Andersch hier auf Zitate von Benjamin Per et zurückgreift. Diese Mischungen und Grenzüberschreitungen können durchaus im Sinne einer Thematisierung der medialen Kommunikation gedeutet werden. Schreibt doch Andersch an anderer Stelle (1977, in Bezug auf Ernst Schnabel):

Das Volk lauscht den Epen, zwar nicht irgend homerischen, aber doch immerhin dem unaufhörlichen Nachrichtenfluß oral vermittelter Historie ${ }^{31}$.

Das Gespräch der Hauptfigur Pierre Grange mit Fayard und Mondello zum Thema Öffentlichkeit verweist indirekt auf die Thematisierung der Wirkungsmöglichkeiten des Hörspiels. Grange glaubt, den Beweis zu haben, daß de Gaulle den Putsch von Algier angezettelt hat, und will eine Liste von Kontaktpersonen in Fayards Blatt veröffentlichen. Fayard lehnt ab, weil die Redaktion soeben beschlossen habe, «de Gaulle eine Chance zu geben», wie es ja die Zeitung Le Monde auch tatsächlich tat. Man bedenke, die Presse als Medium der Öffentlichkeit kapituliert vor etwas, das einer Machtergreifung sehr ähnlich sieht! Der Philosoph Mondello teilt Granges Empörung nicht. Er zweifelt dagegen die Bedeutung von Veröffentlichungen an und zieht die Tat vor. Alles andere sei nichts als Literatur:

Diese Art Literatur - die Literatur als Presse - hat eine gewisse Berechtigung, solange sich die Mächte im Gleichgewicht befinden. Oder wenn die Demokratie sich entwickelt: Zola im Fall Dreyfus. Aber wenn die Politik in den Raum der reinen Macht tritt, ist es aus damit. Dann ist die Presse und ihre Literatur nur noch eine Fußnote zu den Prämissen der Macht. Sie, Pierre, sind ein Opfer gewisser falscher Lehren über die Demokratie. $\mathrm{Zu}$ diesen Irrlehren gehört der Glaube an die Presse und an die unmittelbare Wirkung der Literatur ${ }^{32}$.

Wenn Andersch Mondello so sprechen läßt, dann stellt sich die Frage des Rückbezugs auf die Intention des Hörspiels. Tatsache ist, daß der Autor im Jahre 1958 seine verschiedenen medialen Aktivitäten aufgibt und ins Tessin umsiedelt.

Der Rückzug Anderschs wird häufig in den größeren Zusammenhang der Entwicklung des Rundfunks gestellt. Gegen Ende der fünfziger Jahre kann man immer deutlicher Entwicklungstendenzen konstatieren, die auf die Problematik des Rundfunks bis in die heutige Zeit verweisen. Nach und nach war in den verschiedenen Sendeanstalten der Werbefunk wieder eingeführt worden. Die Gründung des WDR markierte den verstärkten Zugriff der Parteien auf die Kontrollinstanzen des Rundfunks, und zwar nach den Regeln des Proporzes. Das Fernsehen wurde ein immer schärferer Konkurrent des Radios, mit 100000 Teilnehmern im Jahre 1955, aber zwei Jahre später schon mit über einer Million. 1958 begannen die ersten deutschen stereophonen Versuchssendungen, wodurch das Tonband verstärkt vom Aufzeichnungszum Gestaltungsinstrument wurde. Gleichzeitig fingen Kritiker an, von einer Krise des Hörspiels zu reden. Sie äußerte sich durch eine Abnahme der Reflexion des Hörspielgeschehens in den anderen Medien. Hierzu zitiert Klaus Sauer ${ }^{33}$ abermals Andersch:

Das Ausbleiben jeglichen Echos war nicht zu ertragen. In der ersten Hälfte der fünfziger Jahre im Hamburger Sender um Schnabel geschart, haben wir eine Weile geglaubt, eine neue literarische Kunstform kreieren zu können, die begriffen 
werden würde. Aber nur die Hörer haben sie gehört. Das war uns, eitel wie wir sind, zu wenig ${ }^{34}$.

41 Anderschs Abkehr von der Rundfunkarbeit, allerdings nicht vom Hörspiel, wäre demnach nicht so sehr ein Protestakt gegen die Gleichschaltung der Institution durch die Parteien, sondern als Sehnsucht nach literarischem Ruhm zu bewerten, oder aber nach spürbarer Wirksamkeit. Die zaghaften Versuche zur Schaffung einer Gegenöffentlichkeit in den Hörspielen der fünfziger Jahre scheitern letztendlich an der Tatsache, daß das Hörspiel eine Öffentlichkeit ohne Öffentlichkeit bleibt.

Ungeachtet dessen zeigen die hier erwähnten Beispiele drei verschiedene Verbindungen zwischen dem gegenöffentlichen Impuls, der Reflexion des Zeitgeschehens und der Thematisierung der medialen Kommunikation. Von einer bewußten Verzahnung dieser drei Elemente, wie man sie später in einigen Hörspielen des sogenannten Neuen Hörspiels wiederfindet ${ }^{35}$, kann hier zwar noch nicht die Rede sein. Trotzdem zeigen die genannten Beispiele, daß die Entwicklung des Hörspiels der Nachkriegszeit vielleicht doch kontinuierlicher verläuft, als die Verfechter ebendieses Neuen Hörspiels es wahr haben wollten.

\section{NOTES}

1. - Bertolt Brecht: Der Flug der Lindberghs. Musikfestspiele Baden-Baden 1929.

2. - Wolfram Wessels: Das Hörspiel im Dritten Reich. Bonn 1985.

3. - Gerhard Eckert: Das Hörspiel als politische Waffe. In: Berlin hört und sieht, 1940, Nr 33. Man muß dabei bedenken, daß Hörspiele zu der Zeit noch «live» aufgeführt wurden.

4. - Der ehemalige Panzerfunk kompensierte das schlechte Abschneiden Deutschlands beim Verteilen der Mittelwellenlängen auf der Kopenhagener Konferenz von 1948.

5. - Als die bekanntesten und erfolgreichsten unter ihnen wären Wolfgang Weyrauch und Günter Eich zu nennen.

6. - Die Gruppe 47: Sonderband Text + Kritik 1980, Hrg. von Heinz Ludwig Arnold, S. 223.

7. - Nord-Westdeutscher Rundfunk, ab 1955 gespalten in Norddeutscher Rundfunk und Westdeutscher Rundfunk.

8. - Heinz Schwitzke: Das Hörspiel. Dramaturgie und Geschichte. Köln 1963.

9. - Ebd., S. 383.

10. - Reinhard Döhl: Versuch einer Geschichte und Typologie des Hörspiels in Lektionen. Manuskript der Sendungsreihe auf WDR3.

11. - Margaret Bloom: Die westdeutsche Nachkriegszeit im literarischen Originalhörspiel. Frankfurt/ Main 1985.

12. - In den frühen sechziger Jahren schrieb Erasmus Schöfer zum gleichen Thema Der Pikadon.

13. - Otto Heinrich Kühner: Die Übungspatrone. SDR 1950.

14. - Erich Kuby: Hitlers letzte Festung. NWDR 1952.

15. - Schwitzke (Vgl. Anm. 8), S. 324.

16. - Günter Eich: Träume. NWDR 1951.

17. - Reinhard Döhl: Hörspielphilologie? Jahrbuch der Deutschen Schillergesellschaft. Jg 26, 1982. 489-511. 
18. - Günter Eich: Träume. Vier Spiele. Frankfurt/Main 1953.

19. - Hermann Naber: Günter Eich - Fragensteller. Aus Anlaß der Sendung des Hörspiels «Träume» am 26. Januar $1982 \mathrm{im} \mathrm{SFB.}$

20. - Döhl (Vgl. Anm. 17), S. 495.

21. - Eich (Vgl. Anm. 18), S. 142

22. - Marshall Mc Luhan: Understanding Media. Toronto, 1963.

23. - Max Frisch: Kultur als Alibi. In: Max Frisch: Öffentlichkeit als Partner. Frankfurt/Main 1967, S. $15-24$.

24. - Max Frisch: Biedermann und die Brandstifter. In: Spectaculum. Texte moderner Hörspiele. Frankfurt/Main 1963, S. 82.

25. - Ebd.

26. - Ebd.

27. - NWDR 1952.

28. - BR 1955.

29. - In jener Zeit druckten die Editions de Minuit den Sammelband La gengraine mit Zeugenaussagen über Folterungen, die von den französischen Streitkräften in Algerien durchgeführt wurden. Aufgrund seines medizinisch anmutenden Titels wurde die Zensurbehörde hinters Licht geführt.

30. - Alfred Andersch: Die Nacht der Giraffe. In: Fahrerflucht. Hörspiele. München 1965, S. 79.

31. - Alfred Andersch: Die Geheimschreiber. In: A.A.: Öffentlicher Brief an einen sowjetischen Schriftsteller, das Überholte betreffend, S.174. Zitiert nach: Klaus Sauer: Rundfunk und deutsche Gegenwartsgeschichte. In: Deutsche Gegenwartsliteratur. Ausgangsposition und aktuelle Entwicklung. Hrg. von Manfred Durzak. Stuttgart 1981, S. 446.

32. - Andersch (Vgl. Anm. 30), S. 81-82.

33. - Sauer (Vgl. Anm. 31), S. 446.

34. - Andersch (Vgl. Anm. 31), S. 175.

35. - Am deutlichsten vielleicht 1969 in Paul oder die Zerstörung eines Hörbeispiels von Wolf Wondratschek.

\section{RÉSUMÉS}

Im Gegensatz zu den Hörspielen des sogenannten Neuen Hörspiels haben die Hörspiele der fünfziger Jahre nicht den Ruf, Aktualität zu reflektieren, und man hält sie auch insgesamt nicht für besonders kritisch gegenüber der Gesellschaft oder ihrem eigenen Medium. Ausgehend von der Hypothese, daß diese drei Aspekte eines Werks sich gegenseitig bedingen, analysiert dieser Beitrag ihr Zusammenspiel in drei maßgebenden Hörspielen jener Zeit, und versucht zu zeigen, inwiefern sie nicht ans Ende ihrer inneren Logik geführt worden sind.

A la différence des pièces radiophoniques ouest-allemandes de la fin des années 60 , celles des années 50 n'ont pas la réputation de refléter l'actualité, ni d'ailleurs d'adopter des points de vue particulièrement critiques ou de réfléchir leur propre média. Partant de l'hypothèse selon laquelle ces trois aspects sont interdépendants, le présent article analyse leur configuration dans trois œuvres majeures de l'époque, et tente de montrer en quoi ces dernières ne sont pas allées au bout de leur propre logique. 
AUTEUR

JONAS TOPHOVEN

Universität Lille 